\title{
Proof of Renormalizability and Derivation of Dynamical Equations for Relativistic Composite Particle Systems and Reactions in Unified Field Models
}

\author{
H. Stumpf \\ Institut für Theoretische Physik, Universität Tübingen \\ Z. Naturforsch. 36a, 1289-1298 (1981); received October 15, 1981
}

\begin{abstract}
In any quantum field theory of matter, in particular in quark- and subquark models, bound states have to be treated. In coupling theories corresponding bound state equations are derived by the Gell-Mann-Low procedure from the Greenfunction hierarchy. Due to certain presuppositions neither the derivation of such generalized Bethe-Salpeter equations nor the normalization of their amplitudes are selfconsistent. In this paper bound state equations and general reaction equations for composite particles are derived by means of functional techniques which do not rest on such presuppositions. The derivation is performed for a unified lepton-quark model with boson fusion from fermions, which is described by a nonlinear spinorfield equation with higher order derivatives. Besides the removal of infinities, in coupling theories renormalization mainly means the introduction of dressed field operators which allow a biunique map between field operators and particles. For composite particles renormalization means the dressing of the composite systems which must allow the unique identification of dressed composite particle states independently of the interactions which can take place with other composite systems, i.e. this is in principle the same program as in coupling theories but with the treatment of dressed composite particles instead of dressed field operators. The program is performed for the reaction equations mentioned above.
\end{abstract}

The quantum field theoretical description of high energy phenomena requires a proper theoretical treatment of relativistic composite particle systems and their reactions beyond perturbation theory for point particles. In order to perform a theoretical treatment, corresponding dynamical equations for the quantum states of such systems are needed. In conventional relativistic quantum field theory the derivation of such equations was initiated by Salpeter and Bethe [1] who formulated an integral equation for the "wave function" of a relativistic two-particle system. An improved derivation of this equation was given by Gell-Mann and Low [2] who established the connection of the two-particle propagator of the field, i.e. the two-particle Greenfunction, with the bound state wave functions. By this method the derivation of dynamical equations for relativistic composite particle states is reduced to the derivation of equations for the many-particle propagators of the corresponding fields. This approach was initiated by Schwinger [3] who derived equations for Greenfunctions by means of external source operators. In the further development this

Reprint requests to Prof. Dr. H. Stumpf, Institut für Theoret. Physik, Universität Tübingen, Morgenstelle 14, 741 Tübingen. leads to functional equations for corresponding vacuum expectation value functionals, cf. [4]. Besides an extensive discussion of the two-particle equation, in the further development of the theory the multiple-particle structure of the Greenfunctions was studied and equations for three-particle states were derived, cf. Broido [5], Ethofer and Schuck [6]. Concerning reactions, the most interesting quantity is the $S$-matrix. The connection between Greenfunctions and the $S$-matrix was established for point particles by the L.S.Z.-procedure, Lehmann, Symanzik and Zimmermann [7]. An extension of this procedure to composite particles was performed by Huang and Weldon [8]. The rise of quark theories brought about an increasing interest in the theoretical description of relativistic composite particle states and the properties of states, and corresponding equations were thoroughly elaborated in particular on the phenomenological level. The development of a relativistic multi-particle theory along this pattern has, however, a serious drawback. The derivation of dynamical equations for composite particle wave functions as well as the normalization conditions for these wave functions depend upon a spectral decomposition of the many-particle propagators at which a positive definite state space is assumed. In addition, 
an assumption about the pole structure of the propagator is needed. The recent development of relativistic quantum field theory shows that neither the first nor the second assumption will be satisfied in general. On the one side, there is an increasing interest in indefinite metric quantum field theories which by definition violate the first assumption, on the other side, even in conventional quantum field theories negative norms of Salpeter-Bethe wave function appear, Nakanishi [9] which make the method inconsistent, and renormalized propagators exhibit an anomalous pole structure, Fishman and Gersten [10], which contradicts the second assumption. Hence it is obvious that a derivation of dynamical equations for "wave functions" of composite particles is needed which does not rest on both assumptions. In the framework of functional quantum theory which was developed by Stumpf and coworkers, ef. Stumpf [11], several attempts were made to derive such dynamical equations in a more general way, cf. Stumpf [12], without reference to these assumptions. From general considerations of quantum field theory and of functional quantum theory it follows that such equations must fulfill certain conditions in order to provide an appropriate representation of quantum states:

i) The derivation must not rest upon the metric of the state space;

ii) the derivation must not rest upon special pole structure assumptions;

iii) the equations must provide solutions with appropriate boundary conditions; iv) the equations must contain the renormalization of their constituent particles and interactions, i.e. selfenergy and vertex corrections;

v) the equations must satisfy certain integrability conditions;

vi) the equations should reproduce perturbation theoretic results under certain approximations.

In the papers mentioned above [12] it was not possible to satisfy all these conditions simultaneously. In particular conditions iii), v) and vi) were not properly taken into account. In this paper we improve the methods developed so far in such a way as to satisfy all conditions properly. In order to have a definite model we consider a nonlinear spinorfield regularized by dipole ghosts in an indefinite metric state space, which was already used in the previous papers. For the derivation and formulation of the dynamical equations for composite particle states and reactions the techniques of functional quantum theory is used without further references.

\section{General Solution Procedure}

We consider the spinorfield $\psi_{\alpha}(x)$ and its equation

$$
\begin{aligned}
& {\left[\left(-i \gamma^{v} \partial_{\nu}+\mu\right)^{2}\left(-i \gamma^{\varrho} \partial_{\varrho}+m\right)\right]_{\alpha \beta} \psi_{\beta}(x)} \\
& =V_{\alpha \beta \gamma \delta} \psi_{\beta}(x) \bar{\psi}_{\gamma}(x) \psi_{\delta}(x)
\end{aligned}
$$

which was assumed in [13] to establish the basic operator equation of a lepton-quark model. If $\psi_{\alpha}(x)$ and $\bar{\psi}_{\alpha}(x)$ are combined to give a superspinor $\Psi_{\alpha}(x)$, then the corresponding state functionals are defined by

$$
|\mathfrak{T}(j, a)\rangle:=\sum_{n=1}^{\infty} \int\left\langle 0\left|T \Psi_{\alpha_{1}}\left(x_{1}\right) \ldots \Psi_{\alpha_{n}}\left(x_{n}\right)\right| a\right\rangle\left|D_{n}\left(\begin{array}{c}
\alpha_{1} \\
x_{1} \ldots \alpha_{n}
\end{array} x_{n}\right)\right\rangle \mathrm{d}^{4} x_{1} \ldots \mathrm{d}^{4} x_{n}
$$

and have to satisfy the corresponding functional state equation [11], [14]

$$
\left[D_{\alpha \beta}(x) \partial_{\beta}(x)-\hat{V}_{\alpha \beta \gamma \delta} \partial_{\beta}(x) \partial_{\gamma}(x) \partial_{\delta}(x)\right]|\mathfrak{T}(j, a)\rangle=-\Gamma_{\alpha \beta}^{0} A_{\beta \gamma} j_{\gamma}(x)|\mathfrak{T}(j, a)\rangle
$$

where $D_{\alpha \beta}(x)$ represents the superspinor expression of the operator on the left-hand side of (1.1) etc. If (1.3) is projected into the configuration space of the expansion functions of (1.2) it turns out that the right-hand side of (1.3) leads to expressions with singular $\delta$-distributions. In order to secure the integrability of (1.3) these terms must be eliminated by means of a suitable transformation of the state functionals (1.2). This can be achieved in two ways. Either the vacuum functional of the corresponding free field or the vacuum functional of the nonlinear field is split off from the state functional (1.2), i.e. we put

or

$$
|\mathfrak{I}(j, a)\rangle=\exp \left[-\int j_{\alpha}(x) F_{\alpha \beta}\left(x-x^{\prime}\right) j_{\beta}\left(x^{\prime}\right) \mathrm{d}^{4} x \mathrm{~d}^{4} x^{\prime}\right]|\mathfrak{F}(j, a)\rangle
$$

$$
|\mathfrak{T}(j, a)\rangle=\exp [\mathbb{E}(j)]|Z(j, a)\rangle
$$


where $F(x)$ is the two-point function of the free field, while $\mathbb{E}(j)$ is the disconnected vacuum functional of the nonlinear field. Based on similar expansions in classical statistical mechanics, cluster expansions which correspond to the $\widetilde{E}(j)$ functional were introduced in quantum statistical mechanics by Kahn and Uhlenbeck [15]. In quantum field theory such cluster expansions were introduced by Zimmermann [16] who performed a graph-theoretical analysis of perturbation theory. The decomposition of state functionals (1.4), (1.5) was proposed by Symanzik [17]. Rampacher, Stumpf and Wagner [18] applied these transformations in connection with the integrability condition of equation (1.3) mentioned above. A discussion where emphasis is laid on nonlinear spinor theory was given by Dürr and Wagner [19]. For convenience we use only transformation (1.4) for the general proof of renormalizability of the composite particle states with respect to the nonlinear spinorfield (1.1). Transformation (1.5) can be applied equally well but leads to more complicated calculations which run along the same pattern and will be treated elsewhere.

If transformation (1.4) is applied to equation (1.3) we obtain the equation for the $\mathfrak{F}$-functional [13], [14]
$\left[D_{\alpha \beta}(x) \partial_{\beta}(x)-\hat{V}_{\alpha \beta \gamma \delta} \mathrm{d}_{\beta}(x) \mathrm{d}_{\gamma}(x) \mathrm{d}_{\delta}(x)\right]|\mathcal{F}(j, a)\rangle=0$

with

$$
\mathrm{d}_{\beta}(x):=\partial_{\beta}(x)+\int F_{\beta \varkappa}\left(x-x^{\prime}\right) j_{\mathcal{X}}\left(x^{\prime}\right) \mathrm{d}^{4} x^{\prime} .
$$

In [12.III] a general procedure for the solution of a corresponding functional equation for a noncanonical quantized spinorfield was given. This equation differs from (1.6) for a canonical quantized spinorfield formally by only one term. Due to this difference the results of [12.III] cannot directly be taken over for the solution of (1.6). Rather the solution procedure must slightly be modified in order to be applicable to (1.6).

The $\mathfrak{F}$-functional is defined by the expansion

$$
\begin{aligned}
|\mathfrak{F}(j, a)\rangle:= & \sum_{n=1}^{\infty} \int \varphi_{n}\left(x_{\alpha_{1}} \ldots x_{\alpha_{n}} \mid a\right) \\
& \cdot\left|D_{n}\left(\begin{array}{c}
\alpha_{1} \\
x_{1} \ldots x_{n}
\end{array}\right)\right\rangle \mathrm{d}^{4} x_{1} \ldots \mathrm{d}^{4} x_{n} .
\end{aligned}
$$

According to [12.III] we decompose (1.8) into the series

$$
|\mathfrak{F}(j, a)\rangle=\sum_{l=0,2,4, \ldots}^{\infty}\left|\mathfrak{F}_{\varrho+l}(j, a)\right\rangle
$$

where $\varrho$ is a general fermion number of the state $|a\rangle$ for which $\varphi_{n} \equiv 0$ with $n<\varrho$, and obtain from (1.6) the equivalent system of equations

$$
\begin{aligned}
& l=0 ; \quad A_{1}(x)\left|\mathfrak{F}_{\varrho}(j, a)\right\rangle+A_{2}(x)\left|\mathfrak{\mho}_{\varrho+2}(j, a)\right\rangle=0, \\
& l=2 ; \quad A_{1}(x)\left|\mathfrak{F}_{\varrho+2}(j, a)\right\rangle+A_{2}(x)\left|\mathfrak{F}_{\varrho+4}(j, a)\right\rangle+A_{3}(x)\left|\mathfrak{F}_{\varrho}(j, a)\right\rangle=0, \\
& 4 \leqq l<\infty ; \quad A_{1}(x)\left|\mathfrak{F}_{\varrho+l}(j, a)\right\rangle+A_{2}(x)\left|\mathfrak{F}_{\varrho+l+2}(j, a)\right\rangle \\
& +A_{3}(x)\left|\mathfrak{\mho}_{\varrho+l-2}(j, a)\right\rangle+A_{4}(x)\left|\mathfrak{F}_{\varrho+l-4}(j, a)\right\rangle=0
\end{aligned}
$$

with

$$
\begin{aligned}
& A_{1}(x):=D(x) \partial(x)+3 \hat{V} \int F\left(x-x^{\prime}\right) j\left(x^{\prime}\right) \mathrm{d}^{4} x^{\prime} \partial(x) \partial(x), \quad A_{2}(x):=\hat{\nabla} \partial(x) \partial(x) \partial(x), \\
& A_{3}(x):=3 \hat{V} \int F\left(x-x^{\prime}\right) j\left(x^{\prime}\right) \mathrm{d}^{4} x^{\prime} \int F\left(y-y^{\prime}\right) j\left(y^{\prime}\right) \mathrm{d}^{4} y^{\prime} \partial(x), \\
& A_{4}(x):=\hat{V} \int F\left(x-x^{\prime}\right) j\left(x^{\prime}\right) \mathrm{d}^{4} x^{\prime} \int F\left(y-y^{\prime}\right) j\left(y^{\prime}\right) \mathrm{d}^{4} y^{\prime} \int F\left(z-z^{\prime}\right) j\left(z^{\prime}\right) \mathrm{d}^{4} z^{\prime},
\end{aligned}
$$

where spinor indices are suppressed for brevity.

If we define the "polarization cloud" functional $|\chi\rangle$ by

$$
|\chi(j, a)\rangle:=\sum_{l=2,4, \ldots}^{\infty}\left|\mathfrak{F}_{\varrho+l}(j, a)\right\rangle
$$

the system (1.10) can equivalently be expressed by the set of two equations [12.III]

$$
\begin{aligned}
& A_{1}(x)\left|\mathfrak{\mho}_{\varrho}(j, a)\right\rangle+A_{2}(x)|\chi(j, a)\rangle=0, \\
& {[D(x) \partial(x)+\hat{V} \mathrm{~d}(x) \mathrm{d}(x) \mathrm{d}(x)]|\chi(j, a)\rangle=\left[A_{1}(x)+A_{3}(x)+A_{4}(x)\right]\left|\mathfrak{\mho}_{\varrho}(j, a)\right\rangle .}
\end{aligned}
$$

The next step is the elimination of the polarization cloud from (1.14). Due to the difference of the operator $A_{3}$ for canonical and noncanonical quantization this procedure must be modified compared with 
that applied in [12.III]. Application of $G:=D^{-1}$ to the second equation of (1.14) yields

$$
\begin{aligned}
& {\left[\partial(x)+\int G\left(x-x^{\prime}\right) \hat{V} \mathrm{~d}\left(x^{\prime}\right) \mathrm{d}\left(x^{\prime}\right) \mathrm{d}\left(x^{\prime}\right) \mathrm{d}^{4} x^{\prime}\right]|\chi(j, a)\rangle} \\
& =\int G\left(x-x^{\prime}\right)\left[A_{1}\left(x^{\prime}\right)+A_{3}\left(x^{\prime}\right)+A_{4}\left(x^{\prime}\right)\right] \mathrm{d}^{4} x^{\prime}\left|\mathfrak{F}_{\varrho}(j, a)\right\rangle .
\end{aligned}
$$

If the base functionals are defined by

$$
\left|D_{n}\left(z_{1} \ldots z_{n}\right)\right\rangle=\frac{1}{n !} j\left(z_{1}\right) \ldots j\left(z_{n}\right)|0\rangle_{\mathfrak{g}},
$$

where $|0\rangle_{\mathfrak{W}}$ is the functional vacuum state, it can be shown that with

$$
\mathscr{P}(j, \partial):=\sum_{k=1}^{\infty} \int\left|D_{k}\left(z_{1} \ldots z_{k}\right)\right\rangle(k-1) !\left\langle D_{k}\left(z_{1} \ldots z_{k}\right)\right| \mathrm{d}^{4} z_{1} \ldots \mathrm{d}^{4} z_{k}
$$

the relation

$$
\mathscr{P}(j, \partial) \int j(x) \partial(x) \mathrm{d}^{4} x|\chi(j, a)\rangle=|\chi(j, a)\rangle
$$

holds. Application of (1.18) to (1.15) then gives

$$
|\chi(j, a)\rangle=\mathscr{K}(j, \partial)|\chi(j, a)\rangle+\mathscr{R}(j, \partial)\left|\mathfrak{F}_{\varrho}(j, a)\right\rangle
$$

with

$$
\mathscr{K}(j, \partial):=-\mathscr{P}(j, \partial) \int j(x) G\left(x-x^{\prime}\right) \hat{V} \mathrm{~d}\left(x^{\prime}\right) \mathrm{d}\left(x^{\prime}\right) \mathrm{d}\left(x^{\prime}\right) \mathrm{d}^{4} x^{\prime} \mathrm{d}^{4} x
$$

and

$$
\mathscr{R}(j, \partial):=\mathscr{P}(j, \partial) \int j(x) G\left(x-x^{\prime}\right)\left[A_{1}\left(x^{\prime}\right)+A_{3}\left(x^{\prime}\right)+A_{4}\left(x^{\prime}\right)\right] \mathrm{d}^{4} x^{\prime} \mathrm{d}^{4} x .
$$

Equation (1.19) has the iterative solution

$$
|\chi(j, a)\rangle=\sum_{n=0}^{\infty} \mathscr{K}(j, \partial)^{n} \mathscr{R}(j, \partial)\left|\Im_{\varrho}(j, a)\right\rangle .
$$

Substitution of (1.22) into the first equation of (1.14) yields

$$
\left[A_{1}(x)+A_{2}(x) \sum_{n=0}^{\infty} \mathscr{K}(j, \partial)^{n} \mathscr{R}(j, \partial)\right]\left|\mathfrak{F}_{\varrho}(j, a)\right\rangle=0
$$

and this equation has to be solved by nonperturbative methods. As the polarization cloud depends on the decomposition of the $\mathfrak{T}$-functional, this decomposition must be performed in such a way as to minimize the effects of the polarization cloud, i.e. to justify the application of perturbation theory for its elimination.

\section{Symmetry and Boundary Conditions}

The basic functional equation (1.3) of the nonlinear spinorfield $\psi_{\alpha}(x)$ is generated by the application of the nonlinear spinorfield equation (1.1) to one of the constituent field operators of the coefficient functions in (1.2). In addition (1.1) can repeatedly be applied to several constituent field operators of these functions which generates additional functional equations. In the functional formulation such additional equations correspond to the repeated application of the operator of (1.3) to the state functional (1.2). These equations can equivalently be formulated for the transformed functionals of (1.4) or (1.5) etc. For the $\mathfrak{F}$-functional from (1.6) we obtain the additional set of equations

$\prod_{l=1}^{r}\left[D\left(x_{l}\right) \partial\left(x_{l}\right)-\hat{V} \mathrm{~d}\left(x_{l}\right) \mathrm{d}\left(x_{l}\right) \mathrm{d}\left(x_{l}\right)\right]|\mathfrak{F}(j, a)\rangle=0$

for $2 \leqq r<\infty$. These equations must simultaneously be satisfied together with equation (1.6). If we assume that $|\mathfrak{F}(j, a)\rangle$ is a solution of (1.6), then $|\mathfrak{F}(j, a)\rangle$ must also be a solution of (2.1). Hence these equations do not lead to additional conditions with respect to the manifold of solutions of (1.6).

The same procedure can be applied to (1.23) where it proves to be nontrivial. We first write (1.23) in the symbolic form

$$
[D(x) \partial(x)+\mathscr{D}(x, j, \partial)]\left|\mathfrak{F}_{\varrho}(j, a)\right\rangle=0
$$


with

$$
\begin{aligned}
\mathscr{D}(x, j, \partial): & =\int 3 \hat{V} F^{\prime}\left(x-x^{\prime}\right) j\left(x^{\prime}\right) \mathrm{d}^{4} x^{\prime} \mathrm{d}(x) \mathrm{d}(x) \\
& +\hat{V} \partial(x) \partial(x) \partial(x)\left[\sum_{n=0}^{\infty} \mathscr{K}(j, \partial)^{n} \mathscr{R}(j, \partial)\right] .
\end{aligned}
$$

As (1.6) is assumed to be satisfied by the $\mathfrak{F}$-state functional, we need not take into account (2.1). Hence (2.2) is the only equation following from (1.6) and (2.1). But similarly to the original equation, we can also consider the equations which arise from the repeated application of the operator of (2.2) to the $\mathfrak{F}_{\varrho}$-state functional. Then, if we work with an irreducible fermion state in the $\varrho$-sector we obtain the set of equations

$$
\prod_{l=1}^{r}\left[D\left(x_{l}\right) \partial\left(x_{l}\right)+\mathscr{D}\left(x_{l}, j, \partial\right)\right]\left|\mathfrak{F}_{\varrho}(j, a)\right\rangle=0
$$

with $2 \leqq r \leqq \varrho$, since the state functional contains only $\varrho$ coordinates. Repeated application of the operator of (2.2) in the same coordinate need not be considered. The set of (2.4) must simultaneously be satisfied together with (2.2). If we assume that $\left|\mathfrak{F}_{\varrho}(j, a)\right\rangle$ is a solution of $(2.2)$, then it must also be a solution of (2.4). Hence these equations do not lead to additional conditions with respect to the manifold of solutions of (2.2). Nevertheless, (2.4) play an important role with respect to symmetry and boundary conditions.

We first consider (2.2). Since it is formulated in a functional state space derived from anticommuting sources, the expansion coefficient $\varphi_{\varrho}\left(x_{1} \ldots x_{\varrho} \mid a\right)$ of the corresponding state functional $\left|\mathfrak{F}_{\varrho}(j, a)\right\rangle$ must strictly be antisymmetric, i.e. all symmetric terms automatically drop out, resp. vanish. If we apply $D^{-1}$ to (2.2) and project it into configuration space this yields the equation

$$
\begin{aligned}
& \varphi_{\varrho}\left(x_{1}, x_{2} \ldots x_{\varrho} \mid a\right) \\
& =\int G\left(x_{1}-x^{\prime}\right) h\left(x^{\prime}, x_{2} \ldots x_{\varrho}\right) \mathrm{d}^{4} x^{\prime}
\end{aligned}
$$

with

$$
\begin{aligned}
& h\left(x_{1}, x_{2} \ldots x_{\varrho}\right): \\
& =-\left\langle D\left(x_{2} \ldots x_{\varrho}\right)\left|\mathscr{D}\left(x_{1}, j, \partial\right)\right| \mathfrak{\mho}_{\varrho}(j, a)\right\rangle .
\end{aligned}
$$

As (2.5) is the exact equation for the state functional, from the antisymmetry of the left-hand side of (2.5) the antisymmetry of the right-hand side of (2.5) must be concluded. From this it follows that all equations which result from (2.5) by the change $x_{1} \rightarrow x_{i}, x_{i} \rightarrow x_{1}$, i.e.

$$
\begin{aligned}
& D\left(x_{i}\right) \varphi_{\varrho}\left(x_{1} \ldots x_{\varrho} \mid a\right) \\
& =-h\left(x_{i} \ldots x_{1} \ldots x_{\varrho}\right)
\end{aligned}
$$

must be satisfied by the coefficient function $\varphi_{\varrho}\left(x_{1} \ldots x_{\varrho} \mid a\right)$ of the state functional, too, and (2.5) and the set of (2.7) have to be solved under the subsidiary condition of antisymmetry of the $\varphi_{\varrho^{-}}$ functions.

Since it is difficult to solve a set of equations with a subsidiary condition, we try to incorporate this condition directly into (2.2) by deriving from it a symmetry invariant equation which leads to only one equation for the $\varphi_{\varrho}$-function in configuration space. According to [12.II] this can be done either by additive symmetrization or by multiplicative symmetrization. The additive symmetrization was applied in [12.III] but leads to difficulties with the relativistic boundary conditions. Hence we apply the multiplicative symmetrization of [12.II] which is equivalent with the use of

$$
\prod_{l=1}^{\varrho}\left[D\left(x_{l}\right) \partial\left(x_{l}\right)+\mathscr{D}\left(x_{l}, j, \partial\right)\right]\left|\mathfrak{\mho}_{\varrho}(j, a)\right\rangle=0 .
$$

Obviously, this equation is similar to a many-particle Bethe-Salpeter equation, but now derived by a completely different and more general method. It incorporates symmetry and appropriate boundary conditions and if it is used instead of (2.2), then it must be shown that any solution of (2.8) is a solution of (2.2), too. Already Matthews and Salam [20] tried to replace a set of equations of the kind (2.7) by only one single integral equation. But their proof of equivalence is meaningless as in the proof they use relations which they intended to prove. Hence we do not further discuss their approach. Concerning (2.8) the following theorem holds:

Theorem: Suppose any solution of (2.2) resp. (2.8) is antisymmetric, then any solution of (2.8) is also a solution of (2.2) and vice versa.

Proof: If $\varphi_{\varrho}$ is an antisymmetric solution of (2.2), it must be a solution of (2.8), too, as (2.8) is derived from (2.2). If $\varphi_{\varrho}$ is an antisymmetric solution of (2.8) we perform the proof of equivalence in three steps:

i) We first consider the two-fermion case and assume without loss of generality that (2.2) is represented in configuration space by the equation

$$
\left[D\left(x_{1}\right)+A\left(x_{1}-x_{2}\right)\right] \varphi_{2}\left(x_{1}, x_{2}\right)=0 .
$$


In order to abbreviate the notation we map this equation into an appropriate function space defined by the complete orthonormal sets $\left\{\chi_{i}\left(x_{1}\right), \chi_{j}\left(x_{2}\right)\right\}$ where $\varphi(i, j)$ is the map of $\varphi_{2}\left(x_{1}, x_{2}\right)$ etc. If we apply the summation convention, equation (2.9) is represented by

$$
\left[\partial\left(i, i^{\prime}\right) \delta\left(j, j^{\prime}\right)+A\left(i j, i^{\prime} j^{\prime}\right)\right] \varphi\left(i^{\prime}, j^{\prime}\right)=0
$$

with $\delta\left(j, j^{\prime}\right)=\delta_{j j^{\prime}}$. Due to the antisymmetry of $\varphi(i, j)$ from $(2.10)$ the equation

$$
\left[\delta\left(i, i^{\prime}\right) \partial\left(j, j^{\prime}\right)+A\left(j i, j^{\prime} i^{\prime}\right)\right] \varphi\left(i^{\prime}, j^{\prime}\right)=0
$$

can be derived, i.e. must simultaneously hold.

These equations correspond to the set of (2.5) and (2.7). We write them in the symbolic form

$$
\left(\partial_{1}+A\right) \varphi=0
$$

and

$$
\left(\partial_{2}+A^{\mathrm{T}}\right) \varphi=0 .
$$

Then the equation which corresponds to the configuration space representation of (2.8) reads

$$
\left(\partial_{2}+A^{\mathrm{T}}\right)\left(\partial_{1}+A\right) \varphi=0 .
$$

The general solution of (2.14) can be written in the following form

with

$$
\varphi=\left(\partial_{1}+A\right)^{-1} \varphi_{0}+\tilde{\varphi}_{0}
$$

and

$$
\left(\partial_{2}+A^{\mathrm{T}}\right) \varphi_{0}=0
$$

As $\varphi$ is assumed to be antisymmetric, so must be $\tilde{\varphi}_{0}$, and from this it follows that $\tilde{\varphi}_{0}$ has to satisfy (2.13), too. Hence $\tilde{\varphi}_{0}$ is a simultaneous solution of (2.14) as well as of (2.12) (2.13) and we have to investigate only $\left(\partial_{1}+A\right)^{-1} \varphi_{0}$.

We first show by contradiction that $\varphi_{0}$ cannot be antisymmetric. Let $\varphi_{0}$ be antisymmetric. Then from (2.15) we have $\left(\partial_{1}+A\right) \varphi=\varphi_{0}$ for $\tilde{\varphi}_{0}=0$. Due to the antisymmetry we furthermore have the relation $\left(\partial_{2}+A^{\mathrm{T}}\right) \varphi=\varphi_{0}$ and from this by substitution of $\varphi=\left(\partial_{1}+A\right)^{-1} \varphi_{0}$ it follows that $\left(\partial_{2}+A^{\mathrm{T}}\right)\left(\partial_{1}+A\right)^{-1}$ should be the unity operator, which is not true. Hence $\varphi_{0}$ must not be antisymmetric. If it is not antisymmetric, then from $\varphi_{0}=$ $\left(\partial_{1}+A\right) \varphi$ it follows

$$
\begin{aligned}
& \left(\partial_{1}+A\right)\left(i j, i^{\prime} j^{\prime}\right) \varphi\left(i^{\prime} j^{\prime}\right) \\
& =-\left(\partial_{1}+A\right)\left(i j, j^{\prime} i^{\prime}\right) \varphi\left(i^{\prime} j^{\prime}\right) .
\end{aligned}
$$

This relation can be satisfied if

$$
\left(\partial_{1}+A\right)\left(i j, i^{\prime} j^{\prime}\right)=-\left(\partial_{1}+A\right)\left(i j, j^{\prime} i^{\prime}\right)
$$

holds for all $i^{\prime} j^{\prime}$. This is not true. Therefore $\varphi$ must be an antisymmetric solution of (2.12) and of (2.13) and from this we conclude that

$$
\left(\partial_{1}+A\right)\left(\partial_{1}+A\right)^{-1} \varphi_{0}=0 \text {, i.e. } \varphi_{0} \equiv 0 .
$$

Therefore we have $\varphi=\tilde{\varphi}_{0}$.

ii) We consider the $\varrho$-fermion sector. In this case we assume that the initial set of equations for $\varphi_{\varrho}\left(x_{1} \ldots x_{\varrho}\right)$ has the form

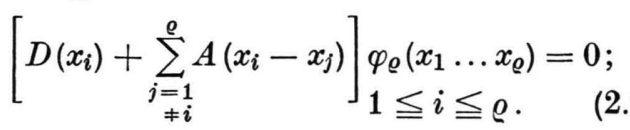

Then the corresponding symmetrized equation reads

$$
\prod_{i=1}^{\varrho}\left[D\left(x_{i}\right)+\sum_{\substack{j=1 \\ \neq i}}^{\varrho} A\left(x_{i}-x_{j}\right)\right] \varphi_{\varrho}\left(x_{1} \ldots x_{\varrho}\right)=0 .
$$

Applying our symbolic notation, we may write for (2.18)

$$
\left(\partial_{i}+A_{i}\right) \varphi_{\varrho}=0 ; \quad 1 \leqq i \leqq \varrho
$$

and for (2.19)

$$
\prod_{i=1}^{\varrho}\left(\partial_{i}+A_{i}\right) \varphi_{\varrho}=0 .
$$

We replace $(2.21)$ by the system

$$
\begin{gathered}
\prod_{i=1}^{\varrho-1}\left(\partial_{i}+A_{i}\right) \varphi_{\varrho}=\varphi_{\varrho}{ }^{0}, \\
\left(\partial_{\varrho}+A_{\varrho}\right) \varphi_{\varrho}=0 .
\end{gathered}
$$

Then any solution of (2.21) is given by

$$
\varphi_{\varrho}=\left[\prod_{i=1}^{e-1}\left(\partial_{i}+A_{i}\right)\right]^{-1} \varphi_{\varrho}^{0}+\tilde{\varphi}_{\varrho}^{0},
$$

where $\tilde{\varphi}_{\varrho}{ }^{0}$ is assumed to be a completely antisymmetric solution of the homogeneous Eq. (2.22). Furthermore we assume that we have demonstrated by the procedure of i) that $\tilde{\varphi}_{\varrho}{ }^{0}$ must also be a solution of $(2.20)$ for $1 \leqq i \leqq \varrho$. Then we have only to discuss the expression

$$
\left[\prod_{i=1}^{0-1}\left(\partial_{i}+A_{i}\right)\right]^{-1} \varphi^{0} .
$$

But by the procedure of i) it can be shown that $\varphi_{e}{ }^{0}$ must vanish. Hence $\varphi_{\varrho} \equiv \tilde{\varphi}_{\varrho}{ }^{0}$ and we have verified the theorem also for $\varrho$-fermion sector.

iii) Finally, we observe that the procedure of i) can also be applied to more complicated interac- 
tions, since it is essential that $\varphi_{\varrho}$ satisfies a set of equations of the kind (2.19) but not that we use some special kind of interactions, Q.E.D.

Having shown the equivalence of (2.2) and (2.8) we can analyze the structure of (2.8). For brevity we consider only the case $\varrho=2$, where we can already study the problem of boundary conditions. Due to the above theorem we may use Eq. (2.2) for a reformulation of (2.8). Equations (2.2) and (2.8) read for $\varrho=2$

$$
\begin{aligned}
& {\left[D\left(x_{i}\right) \partial\left(x_{i}\right)+\mathscr{D}\left(x_{i}, j, \partial\right)\right]\left|\widetilde{J}_{2}(j, a)\right\rangle=0 ;} \\
& i=1,2
\end{aligned}
$$

and

$$
\prod_{i=1}^{2}\left[D\left(x_{i}\right) \partial\left(x_{i}\right)+\mathscr{D}\left(x_{i}, j, \partial\right)\right]\left|\mho_{2}(j, a)\right\rangle=0
$$

Evaluation of (2.26) yields

$$
\begin{aligned}
\left\{D\left(x_{1}\right) \partial\left(x_{1}\right) D\left(x_{2}\right) \partial\left(x_{2}\right)\right. & +\mathscr{D}\left(x_{1}, j, \partial\right) D\left(x_{2}\right) \partial\left(x_{2}\right)+\mathscr{D}\left(x_{2}, j, \partial\right) D\left(x_{1}\right) \partial\left(x_{1}\right) \\
& \left.+\left[D\left(x_{1}\right) \partial\left(x_{1}\right), \mathscr{D}\left(x_{2}, j, \partial\right)\right]_{-}+\mathscr{D}\left(x_{1}, j, \partial\right) \mathscr{D}\left(x_{2}, j, \partial\right)\right\}\left|\widetilde{\mho}_{2}(j, a)\right\rangle=0 .
\end{aligned}
$$

If now (2.25) is used we obtain the equation

$$
\left\{D\left(x_{1}\right) D\left(x_{2}\right) \partial\left(x_{1}\right) \partial\left(x_{2}\right)+\left[D\left(x_{1}\right) \partial\left(x_{1}\right), \mathscr{D}\left(x_{2}, j, \partial\right)\right]_{-}-\mathscr{D}\left(x_{2}, j, \partial\right) \mathscr{D}\left(x_{1}, j, \partial\right)\right\}\left|\mathfrak{\mho}_{2}\right\rangle=0 .
$$

The commutator in (2.28) plays the role of a general potential which has to be added to the $\mathscr{D}^{2}$ potential term. Hence if we invert the differential operators of the first term we obtain a boundary value structure which corresponds to that of a Bethe-Salpeter equation and which is required for a proper $S$-matrix calculation by means of the L.S.Z.-reduction technique. The discussion of the boundary values is so far restricted to the basic fermions of the theory. In the next section we will include composite particle states in the calculation procedure.

\section{Proof of Renormalizability}

In renormalizable quantum field theories with divergent terms, renormalization serves for the removal of these divergencies by counterterms added to the Lagrangian of the original field. But renormalization still has another even more fundamental task which is valid independently whether a quantum field theory possesses divergencies or not. If the field operators of such a quantum field are assumed to describe physical particles, then those field operators which occur in the unrenormalized original Lagrangian (bare field operators) can have no physical interpretation. Since their corresponding bare mass-values are corrected by selfenergy terms which cause mass-shifts, the corresponding physical particles are located at another mass-shell than the bare "particle" massshell. Hence in order to get a unique description of the physical particles by field operators, the bare field operators must be converted into dressed field operators which just contain these mass-shifts and whose introduction avoids discrepancies between theory and experiment and allows a biunique relation between particles and fields. Hence the introduction of dressed field operators must be a common feature of all field theories. Via the asymptotic conditions such dressed fields guarantee that the theory asymptotically contains only definite kinds of particles so that by calculating the effects of interactions in various orders, the physical content of the theory with respect to its in- resp. out-states is not changed. This invariance of the physical content of a theory under approximations allows a physical interpretation of its results which is independent of approximations used. Hence this invariance is an essential ingredient of any meaningful field theory and must be established at the very beginning of its investigation.

For composite particle theories of the kind considered here the field operators lose their physical meaning, but nevertheless the question remains: are there particles which can be defined independently of the various approximations? The proof of renormalizability in composite particle theories is the procedure to answer this problem. If the theory shows infinities, the first step in this procedure must be the introduction of renormalized field operators, although in this case they lack a physical interpretation. In the case of equation (1.1) the dimension of the field operators is $\left[\mathrm{cm}^{-1 / 2}\right]$ and that of the coupling constant $\left[\mathrm{cm}^{-2}\right]$ hence no infinities can occur in single diagrams. Hence we need not introduce renormalized field operators in 
a first step. Rather we can immediately treat the dressed particle problem.

According to the preceding section the quantized field of (1.1) produces a corresponding linear state space whose functional representation is characterized by the set of (2.8) for all meaningful $\varrho$-values. The proof of renormalization then consists in the investigation whether these equations admit a unique definition of composite particles or not; i.e. the same set of composite particles must theoretically be recovered in any asymptotic configuration which may be derived from the set of (2.8). A proof of this renormalizability for composite particles was first given by Stumpf [12.III] who treated the additive symmetrized functional equations. An attempt was made in [12.II] to show renormalizability for the multiplicative symmetrization. We are interested only in the renormalization of the multiplicative symmetrized equations and give an improved elaboration of the approach in [12.II].

We first consider the set of (2.8) with the fermion numbers $\left\{\varrho_{i}, 1 \leqq i<\infty\right\}$ which lead to the formation of bound states, i.e. to composite particles. By direct calculation it follows from (2.8) that the corresponding bound state equations for the wave functions $\varphi_{e_{i}}\left(x_{1} \ldots x_{e_{i}} \mid a_{i}\right)$ must be given by the projections

$$
\left\langle 0\left|\mathfrak{\complement}_{\varrho_{i}}\left(x_{1} \ldots x_{\varrho_{i}}, j, \partial\right)\right| \mathfrak{F}_{\varrho_{i}}\left(j, a_{i}\right)\right\rangle=0 ; \quad\left\{\varrho_{i}, 1 \leqq i<\infty\right\}
$$

with

$$
\left|\mathfrak{\mho}_{Q_{i}}\left(j, a_{i}\right)\right\rangle:=\int \varphi_{\varrho_{i}}\left(x_{1}^{\prime} \ldots x_{\varrho_{i}}^{\prime} \mid a_{i}\right)\left|D\left(x_{1}^{\prime} \ldots x_{\Theta_{i}}^{\prime}\right)\right\rangle \mathrm{d}^{4} x_{1} \ldots \mathrm{d}^{4} x_{\varrho_{s}}
$$

and

$$
\mathfrak{V}_{Q_{l}}\left(x_{1} \ldots x_{Q_{i}}\right):=\prod_{l=1}^{e_{i}}\left[D\left(x_{l}\right) \partial\left(x_{l}\right)+\mathscr{D}\left(x_{l}, j, \partial\right)\right] .
$$

Equation (3.1) can be rewritten in the form

$$
\begin{aligned}
& \int\left\langle 0\left|j\left(x_{1}^{\prime}\right) \ldots j\left(x_{Q_{i}}^{\prime}\right) \mathbb{C}_{\varrho_{i}}\left(x_{1} \ldots x_{\varrho_{i}}, j, \partial\right)\right| 0\right\rangle \varphi_{Q_{i}}\left(x_{1}^{\prime} \ldots x_{\varrho_{i}}^{\prime} \mid a_{i}\right) \mathrm{d}^{4} x_{1}^{\prime} \ldots \mathrm{d}^{4} x_{Q_{i}}^{\prime}
\end{aligned}
$$

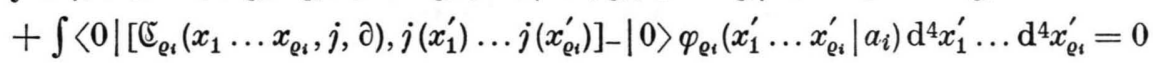

and since $\langle 0| j(x)=0$, the first term of (3.4) vanishes. If the remaining commutator is evaluated and applied to the functional vacuum state, then due to $\partial(x)|0\rangle=0$ this leads to a series expansion

$$
\left[\mathbb{C}_{Q_{i}}\left(x_{1} \ldots x_{Q_{i}}, j, \partial\right), j\left(x_{1}^{\prime}\right) \ldots j\left(x_{Q_{i}}^{\prime}\right)\right]-|0\rangle=C_{Q_{i}}^{0}\left(x_{1} \ldots x_{Q_{i}} \mid x_{1}^{\prime} \ldots x_{Q_{i}}^{\prime}\right)|0\rangle+\sum_{n=1}^{\infty} C_{Q_{i}}^{n}\left|D_{n}\right\rangle,
$$

where the expansion coefficients $C_{\varrho_{i}}^{n}$ for $n \geqq 1$ are only symbolically written. With (3.5) Eq. (3.4) yields

$$
\int C_{\varrho_{i}}^{0}\left(x_{1} \ldots x_{\varrho_{i}} \mid x_{1}^{\prime} \ldots x_{\varrho_{i}}^{\prime}\right) \varphi_{\varrho_{i}}\left(x_{1}^{\prime} \ldots x_{\varrho_{i}}^{\prime} \mid a_{i}\right) \mathrm{d}^{4} x_{1}^{\prime} \ldots \mathrm{d}^{4} x_{\varrho_{i}}^{\prime}=0 .
$$

For the treatment of the general Eq. (2.8) we assume that $\varrho$ allows the partition $\varrho=\sum_{i=1}^{k} \varrho_{\alpha_{i}}$ where $\varrho_{\alpha_{i}}$ is an element of the set $\left\{\varrho_{i}\right\}$. According to [12.II] we introduce an intermediate antisymmetry breaking by defining the following decomposition

$$
\left(x_{1} \ldots x_{\varrho}\right)=\left(x_{1} \ldots x_{z_{1}}\left|x_{z_{1}+1} \ldots x_{z_{2}}\right| \ldots \mid x_{z_{k-1}+1} \ldots x_{z_{k}}\right)
$$

with $z_{i}=\sum_{j=1}^{i} \varrho_{\alpha_{i}}$. Then the operator of (2.8) admits the representation

$$
\mathfrak{\complement}_{\varrho}\left(x_{1} \ldots x_{\varrho}, j, \partial\right)=\prod_{l=1}^{k} \complement_{\varrho_{\alpha_{l}}}\left(x_{z_{l-1}+1} \ldots x_{z_{l}}, j, \partial\right)
$$

and (2.8) can equivalently be expressed by

$$
\int\left\langle 0\left|\prod_{l=1}^{k} \complement_{\varrho_{l}}\left(x_{z_{l-1}+1} \ldots x_{z_{l}}, j, \partial\right) \prod_{l=1}^{k} j\left(x_{z_{l-1}+1}^{\prime}\right) \ldots j\left(x_{z_{l}}\right)\right| 0\right\rangle \varphi_{\varrho}\left(x_{1}^{\prime} \ldots x_{z_{1}}^{\prime}|\ldots| x_{z_{k-1}+1}^{\prime} \ldots x_{z_{k}}^{\prime}\right) \mathrm{d}^{4} x_{1}^{\prime} \ldots \mathrm{d} 4 x_{\varrho}^{\prime}=0 \text {. }
$$


By repeated application of commutators, this equation can be rearranged to give

$$
\begin{aligned}
& \int\left\langle 0\left|\prod_{l=1}^{k}\left[\complement_{\varrho_{l}}\left(x_{z_{l-1}+1} \ldots x_{z_{l}}, j, \partial\right), j\left(x_{z_{l-1}+1}^{\prime}\right) \ldots j\left(x_{z_{l}}^{\prime}\right)\right]-\right| 0\right\rangle \varphi_{\varrho}\left(x_{1}^{\prime} \ldots x_{z_{1}}^{\prime}|\ldots| x_{z_{k-1}+1}^{\prime} \ldots x_{z_{k}}^{\prime}\right) \mathrm{d}^{4} x_{1}^{\prime} \ldots \mathrm{d}^{4} x_{\varrho}^{\prime} \\
& +\int\left\langle 0\left|\mathscr{W}\left(x_{1} \ldots x_{z_{1}}|\ldots| x_{z_{k-1}+1} \ldots x_{z_{k}}\left|x_{1}^{\prime} \ldots x_{z_{1}}^{\prime}\right| \ldots \mid x_{z_{k-1}+1}^{\prime} \ldots x_{z_{k}}^{\prime}, j, \partial\right)\right| 0\right\rangle \\
& \cdot \varphi_{\varrho}\left(x_{1}^{\prime} \ldots x_{z_{1}}^{\prime}|\ldots| x_{z_{k-1}+1}^{\prime} \ldots x_{z_{k}}^{\prime}\right) \mathrm{d}^{4} x_{1}^{\prime} \ldots \mathrm{d}^{4} x_{\varrho}^{\prime}=0
\end{aligned}
$$

where the $\mathscr{W}$-functional contains those terms which arise by extracting the commutator term from $\mathfrak{E}_{\varrho}$. For a general investigation it is not necessary to derive the explicit form of $\mathscr{W}$, but it has to be emphasized that $\mathscr{W}$ can uniquely be calculated once $\mathfrak{C}_{\varrho}$ is given. Similarly the commutator terms of (3.10) can exactly be calculated. But for the general investigation it is sufficient to know that the commutator term of (3.10) has the decomposition

$$
\begin{aligned}
& \left\langle 0\left|\prod_{l=1}^{k}\left[\complement_{\varrho_{\alpha_{l}}}\left(x_{z_{l-1}+1} \ldots x_{z_{l}}, j, \partial\right), j\left(x_{z_{l-1}+1}^{\prime}\right) \ldots j\left(x_{z_{l}}^{\prime}\right)\right]-\right| 0\right\rangle \\
& =\prod_{l=1}^{k} C_{\varrho_{\alpha_{l}}}^{0}\left(x_{z_{l-1}+1} \ldots x_{z_{l}} \mid x_{z_{l-1}+1}^{\prime} \ldots x_{z_{l}}^{\prime}\right)+\tilde{W}\left(x_{1} \ldots x_{\varrho} \mid x_{1}^{\prime} \ldots x_{\varrho}^{\prime}\right)
\end{aligned}
$$

where the first term contains the contribution of the composite particle integral kernels without any interaction, while the second term arises from the mutual influence of the commutators and must be interpreted as an additional interaction between the composite particles. The whole Eq. (3.10) then reads

$$
\begin{aligned}
& \int\left[\prod_{l=1}^{k} C_{\varrho_{\alpha_{l}}}^{0}\left(x_{z_{l-1}+1} \ldots x_{z_{l}} \mid x_{z_{l-1}+1}^{\prime} \ldots x_{z_{l}}^{\prime}\right)+\tilde{W}\left(x_{1} \ldots x_{\varrho} \mid x_{1}^{\prime} \ldots x_{\varrho}^{\prime}\right)+W\left(x_{1} \ldots x_{\varrho} \mid x_{1}^{\prime} \ldots x_{\varrho}^{\prime}\right)\right] \\
& \cdot \varphi_{\varrho}\left(x_{1}^{\prime} \ldots x_{z_{1}}^{\prime}|\ldots| x_{z_{k-1}+1}^{\prime} \ldots x_{z_{k}}^{\prime}\right) \mathrm{d}^{4} x_{1}^{\prime} \ldots \mathrm{d}^{4} x_{\varrho}^{\prime}=0 .
\end{aligned}
$$

Application of the product of resolvents $\prod_{l=1}^{k} C_{e_{\alpha_{l}}}^{0-1}$ to (3.12) leads to an inhomogeneous term which describes a set of free ingoing resp. outgoing composite particles and which is a solution of (3.12) for $\tilde{W}=W=0$. In a symbolic notation this yields

$$
\varphi_{\varrho}=\prod_{l=1}^{k} C_{\varrho_{\alpha_{l}}^{0-1}}^{0}(\tilde{W}+W) \varphi_{\varrho}+\prod_{l=1}^{k} \varphi_{\varrho_{\alpha_{l}}} .
$$

The resulting solution $\varphi_{\varrho}$ is not antisymmetric. The subsequent antisymmetrization was already discussed in [12.II] and need not be repeated here.

As the rearrangement of (2.8) is valid for any $\varrho$ and as we have shown that the interaction free term $\prod_{l=1}^{k} C_{e_{\alpha_{l}}}^{0}$ always contains integral kernels which

[1] E. Salpeter and H. Bethe, Phys. Rev. 84, 1232 (1951).

[2] M. Gell-Mann and F. Low, Phys. Rev. 84, 350 (1951).

[3] J. Schwinger, Proc. Nat. Acad. Sci. 37, 432 (1951).

[4] D. Lurie: Particles and Fields, Interscience Publ., New York 1968, Chapter X.

[5] M. Broido, Rep. Progr. Theor. Phys. 32, 493 (1969).

[6] S. Ethofer and P. Schuck, Z. Physik 228, 264 (1969).

[7] H. Lehmann, K. Symanzik, and W. Zimmermann, Nuovo Cim. 1, 205 (1955). belong to the same set of composite particle states, we have demonstrated the renormalizability of the theory defined in the sense above.

This result, however, needs further improvement. For a complete proof it has to be verified that the definition of the composite particle states is independent of the kind of transformation (1.4) resp. (1.5) etc. A successful solution of this problem would include the proof of existence of solutions of Eq. (1.3) which is far beyond the scope of present day mathematics. Therefore, in treating physical problems, any calculation must be done with respect to the same transformation in order to obtain consistent results.

[8] K. Huang and H. Weldon, Phys. Rev. D 11, 257 (1975).

[9] N. Nakanishi, Prog. Theor. Phys. Suppl. 51, 1 (1972).

[10] S. Fishman and A. Gersten, Phys. Rev. 13, 1769 (1976); Phys. Rev. 14, 2162 (1976).

[11] H. Stumpf, Ann. Phys. Germ. 13, 294 (1964); H. Stumpf, Funktionale Quantentheorie, in: Quanten und Felder, ed. H. P. Dürr, Vieweg-Verlag, Braunschweig 1971; H. Stumpf, Acta Phys. Austriaca 
Suppl. IX, 195 (1972); H. Stumpf, New representation spaces of the Poincaré group and functional quantum theory, in: Groups, Systems, and Many-Body Physics, ed. P. Kramer and M. Dal Cin, Vieweg-Verlag, Braunschweig 1980.

[12] I. H. Stumpf, Z. Naturforsch. 26a, 623 (1971). II. H. Stumpf, Z. Naturforsch. 29 a, 549 (1974). III. H. Stumpf, Z. Naturforsch. 31 a, 1361 (1976).

[13] H. Stumpf, Z. Naturforsch. 35a, 1289 (1980); Z. Naturforsch. 36a, 261 (1981).
[14] T. Pouradjam, Thesis, University of Tübingen 1980.

[15] B. Kahn and G. Uhlenbeck, Physica 5, 399 (1938).

[16] W. Zimmermann, Nuovo Cim. 11, 43 (1954).

[17] K. Symanzik, Z. Naturforsch. 9a, 809 (1954).

[18] H. Rampacher, H. Stumpf, and F. Wagner, Fortschr. Phys. 13, 385 (1965).

[19] H. Dürr and F. Wagner, Nuovo Cim. 46, 223 (1966).

[20] P. Matthews and A. Salam, Proc. Roy. Soc. London A 221, 128 (1954). 